survival rates to quote to patients for different treatment modalities.

It follows a logical progression to the pre-surgical planning stages, with detailed analysis on the planning procedure for successful functional implants, taking into account factors such as periodontal and systemic diseases. The presurgical planning chapters give an insight into the complexity and importance of correctly placing implants, giving the reader an appreciation of the depth of knowledge needed to complete this pivitol stage successfully. Presurgical interventions are covered in depth which shows the reader what is possible where first it seems impossible to use implant retained restorations. The influence of modern technology on implant dentistry is very apparent here, and the many technologies in the armamentarium of the implantologist are explored in depth. These sections, as well as the following sections on the surgical placement phase are well illustrated with good quality colour photographs.

The mechanism and biology of osseointegration and the response of the tissues is comprehensively covered over three chapters. This section is quite scientific, is quite taxing to read and is certainly to a postgraduate standard.

The restorative portion focuses more on the planning, timing and loading protocols as opposed to the prosthodontic restoration. This section doesn't prepare the clinician for the clinical stages of providing the restoration such as impression protocols, occlusion, ceramic materials etc. It helps the clinician plan this phase, but not execute it.

In summary this is a well written, well organised and well illustrated textbook which combines the knowledge and experience of the leading figures in implantology. It is a worthwhile read for any implantologist while also being a good read for the general dental practitioner, to whom it gives an understanding of the sequence of events from planning to restoration, enabling then to recognise what is possible for their patients and enable them to coherently explain these treatment options prior to referring to an implantologist.

G. Quilligan

\section{INTRODUCTION TO METAL CERAMIC TECHNOLOGY, 2ND EDITION}

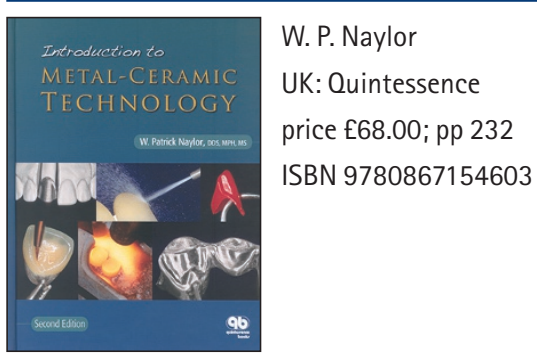

This text aims to introduce a skill-orientated technical approach to metalceramics in practice. Each chapter has been revised with the aim of providing detailed technical material data from the original text, and is complemented throughout by contemporary product information.

The history of the metal-ceramic restoration is described and expanded upon to enlighten the reader on the evolution of the interface between these materials. The chemistry of porcelain is explored and includes the contemporary classifications of this material. In addition, subjects such as fluorescence, metamerism, and opalescence are explained and clarified along with other designations of the material to reflect current usage. Various alloys are included in the discussion, and a section on biocompatibility has been expanded to highlight the indications of allergic responses to the constituent elements.

The fundamentals of spruing, investing, and casting are described, discussed and categorised to allow the reader a true understanding of their principles and aims. The classical and contemporary mechanisms of the porcelain-metal attachment and substructure preparation are covered in depth and include the reasons for bond failure.

Following this, the application of the porcelain to the metal, buildup techniques and firing are covered. Finally, the adjusting and finishing of the restoration and the colour science interface are explained.

For any practising dental practitioner, a thorough understanding of a materials properties and limitations are crucial for case selection, treatment planning, and ultimately, success. This book expertly guides the reader through the world of ceramic-metal technology in a clinically relevant manner. Although at times detailed beyond the requirements of the average practitioner, the structure and presentation of salient points, and the rationale behind methodologies cannot fail to be of use to all dental surgeons. All aspects of the text are beautifully illustrated to ensure maximum impact and, of particular note, at the close of each chapter are synopses of selected journal articles and textbooks for further study and evidence based learning.

This book is highly recommended for any practitioner wishing to gain more from their materials and a must for any dental institute's library.

M. Brennand Roper

\section{OPEN WIDE! A SERIES \\ OF EIGHTEENTH AND NINETEENTH CENTURY CARICATURES ON DENTISTRY}

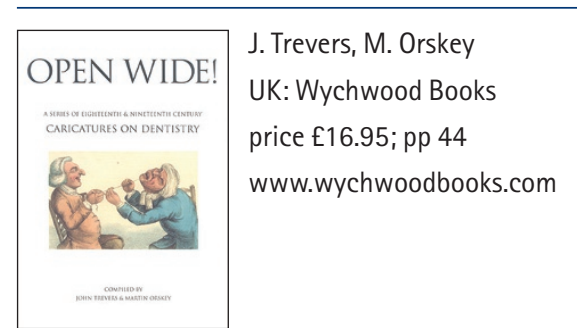

This nicely-produced book is only 44 pages in length. Its 18 paintings and other pictures illustrate a variety of amusing dental situations including toothache and tooth extractions - not that the patients shown would necessarily find them so. Also shown are tooth transplants and the use of an electric generating machine to relieve muscle pain. Both city and country dental practitioners are shown. Amongst the great eighteenth and nineteenth century cartoonists represented are works by George Cruikshank, Thomas Rowlandson and James Gillray. The text opposite each picture briefly but adequately describes for the reader what is being shown.

The authors are to be congratulated on this short, fun-filled book which brings together cartoons with a dental theme. It deserves a place on every coffee and waiting room table. Hopefully it will make relatives, friends and patients aware of how lucky today's patients are.

S. Gelbier 